\section{Prevalência de sibilância e fatores associados em crianças menores de 5 anos de Cuiabá, Mato Grosso, Brasil}

\author{
Prevalence of wheezing and associated factors \\ in children under 5 years of age in Cuiabá, \\ Mato Grosso State, Brazil
}

\section{Prevalencia y factores asociados con sibilancias en niños menores de 5 años en Cuiabá, Mato Grosso, Brasil}

Antonia Maria Rosa 1,2

Ludmilla da Silva Viana Jacobson 3,4

Clóvis Botelho 2,5

Eliane Ignotti 1,2

\footnotetext{
${ }_{1}^{1}$ Faculdade de Ciências da Saúde, Universidade do Estado de Mato Grosso, Cáceres, Brasil.

2 Programa de Pós-graduação em Ciências da Saúde, Universidade Federal de Mato Grosso, Cuiabá, Brasil. ${ }^{3}$ Instituto de Matemática e Estatística, Universidade Federal Fluminense, Niterói Brasil.

${ }^{4}$ Instituto de Medicina Social, Universidade do Estado do Rio de Janeiro, Rio de Janeiro, Brasil. 5 Faculdade de Medicina, Universidade Federal de Mato Grosso, Cuiabá, Brasil.

Correspondência A. M. Rosa

Faculdade de Ciências da Saúde, Universidade do Estado de Mato Grosso.

Av. Getúlio Vargas 2335, Cáceres, MT 78200-000, Brasil. antonia-mr@unemat.br
}

\begin{abstract}
Wheezing is highly prevalent in Brazil, with variations in rates and risk factors between the regions of the country. This cross-sectional study aimed to analyze the prevalence of wheezing in children under 5 years of age $(n=733)$ in Cuiabá, Mato Grosso State, Brazil. The study used a brief version of the standardized instrument from the International Study of Wheezing in Infants (EISL). Analysis of factors associated used logistic regression with a hierarchical approach. Prevalence of wheezing in the previous 12 months was $43.2 \%$. Factors associated with wheezing were: lack of at least 6 months of breastfeeding (adjusted OR = 1.91; 95\%CI: 1.18-3.06), diagnosis of asthma in the family (adjusted OR= 2.02; 95\%CI: 1.06-3.87), current respiratory infection (adjusted OR = 1.81; 95\%CI: 1.05-3.14), and male gender (adjusted $O R=1.50 ; 95 \% C I$ : 1.07-2.11). The study concludes that prevalence of wheezing is high among children in this age bracket in Cuiabá and is associated with factors related to allergy, current respiratory infection, and male gender.
\end{abstract}

Wheezing; Asthma; Child

\section{Resumo}

A prevalência de sibilância no Brasil é elevada, com variações entre as regiões do país, bem como com fatores de risco diferenciados. Com o objetivo de analisar a prevalência de sibilância e fatores associados em menores de 5 anos em Cuiabá, Mato Grosso, Brasil, realizou-se estudo transversal, cuja amostra constou de 733 crianças. Foi utilizado o instrumento padronizado, resumido do Estudio Internacional de Sibilancias en Lactantes (EISL). Para análise dos fatores associados à sibilância foi aplicada regressão logística com abordagem hierarquizada. A prevalência de sibilância nos últimos 12 meses foi de 43,2\%. Foram associados à sibilância: não amamentação ao seio por 6 meses ou mais (OR ajustada =1,91; IC95\%: 1,18-3,06), diagnóstico de asma familiar (OR ajustada = 2,02; IC95\%: 1,06-3,87), doença prévia (OR ajustada =1,81; IC95\%: 1,05-3,14) e sexo masculino (OR ajustada $=1,50$; IC95\%: 1,07-2,11). Concluiu-se que a prevalência de sibilância em crianças dessa faixa etária em Cuiabá é elevada e está associada a fatores relacionados à provável atopia, doença prévia e sexo masculino.

Sibilo; Asma; Criança 


\section{Introdução}

Estudos têm revelado elevada prevalência de sibilância e subdiagnóstico de asma 1,2,3,4. No atendimento clínico, os diversos subgrupos etários apresentam diferentes padrões de sintomas de asma, tratamento e utilização de serviços de saúde 5 .

A sibilância é um sinal que acomete os indivíduos em razão de doenças diversas, tais como refluxo gastroesofágico, doenças respiratórias infecciosas e asma. Assim, possivelmente os casos de sibilância sejam muito mais frequentes na população infantil que casos de asma propriamente ditos. Entretanto, a asma constitui-se na doença crônica mais prevalente em crianças, com implicações na qualidade de vida das mesmas e de suas famílias 6 .

A asma é uma doença inflamatória crônica associada à hiper-responsividade das vias aéreas com consequentes episódios recorrentes de sibilos, dispneia, opressão torácica e tosse, especialmente à noite ou no início da manhã 6,7 . Pode ser de origem atópica (inflamação eosinofílica) ou não atópica (inflamação neutrofílica) ${ }^{8}$. Essa origem dual da asma também pode estar relacionada a fatores de risco diferentes para deflagração dos casos de sibilância entre os grupos 9. Como exemplo, história familiar de asma e infecções respiratórias no início da infância aumentaram o risco de sibilância em crianças atópicas e não atópicas. Em não atópicos, os fatores associados à sibilância foram: pobreza, infecções, baixo nível educacional materno, limpeza do quarto deficiente, infestação de roedores e frequentar creches 10 .

A mensuração dos casos de asma em estudos epidemiológicos apresenta inúmeros desafios especialmente em razão da ausência de um padrão-ouro. Em pesquisas populacionais, o uso de questionário padronizado tem sido cada vez mais utilizado, especialmente como forma de screening dos casos. No Brasil, Bianca et al. 2 e Chong Neto \& Rosário 11 validaram e expandiram para a faixa etária de até 36 meses de vida o instrumento utilizado pelo Estudio Internacional de Sibilancias en Lactantes (EISL) 12. Originalmente proposto para lactentes de 12 a 15 meses de vida 12 , esse instrumento mostrou-se sensível (83,3\%), específico (88,5\%), com valor preditivo positivo de $82 \%$, valor preditivo negativo de $89,3 \%$ e acurácia de $86,5 \%$ para as crianças de até 36 meses, o que o torna um instrumento apropriado para identificação dos casos de sibilância nessa faixa etária 2 .

Dada a importância do conhecimento da prevalência de sibilância e dos sintomas de asma, bem como da inexistência de estudo de base populacional sobre esta doença na faixa etária de menores de 5 anos no estado, delineou-se este estudo. O objetivo é analisar a prevalência de sintomas de asma e os fatores relacionados em menores de 5 anos de idade em Cuiabá, Mato Grosso.

\section{Material e métodos}

\section{População e área do estudo}

Estudo transversal, de base populacional, realizado em Cuiabá, capital do Estado de Mato Grosso. O município possui uma extensão territorial de $3.362 .755 \mathrm{~km}^{2}$ e um clima tropical quente e úmido. Segundo dados do último censo populacional do Instituto Brasileiro de Geografia e Estatística (IBGE.Cidades@. http://www.ibge.gov. $\mathrm{br} /$ cidadesat/topwindow.htm? 1 , acessado em 20/Out/2011), Cuiabá possui 551.098 habitantes, dos quais mais de $90 \%$ residentes na área urbana. A população menor de 5 anos de idade no município é de 40.619 habitantes. Desses, 20.835 (51,3\%) são do sexo masculino e 19.784 (48,7\%) do feminino. Em relação à distribuição por faixa etária, $8.008(19,7 \%)$ crianças são menores de 1 ano e 32.545 (80,3\%) de 1 a 4 anos.

Optou-se por realizar a presente pesquisa em Cuiabá por concentrar um grande contingente populacional, dispor de uma rede de serviços que oferece atendimento em todos os níveis de complexidade e concentrar a maior parte dos pneumologistas do estado.

Estimou-se o tamanho da amostra considerando uma precisão de $5 \%$, um poder de $80 \%$, nível de 95\% de confiança e prevalência de sibilância na literatura entre $10 \%$ e $45,5 \%$. Assim, o tamanho da amostra foi definido em 591 crianças. A esse valor, acrescentou-se 25\% para compensar as possíveis perdas, totalizando 739 crianças.

Foi definido um plano amostral em dois estágios com base nos 653 setores censitários delimitados pelo IBGE. Inicialmente, foi definido o tamanho da amostra de cada setor $(\mathrm{n} i=20)$ e a partir deste número definiu-se o de setores censitários $(n=37)$. A seleção dos setores foi feita por meio de amostragem sistemática.

Com base na malha urbana fornecida pelo IBGE, subseção de Cuiabá, com as delimitações dos setores censitários, definiu-se que o início dos trabalhos seria realizado sempre pela rua inicial de demarcação do setor, quadra à direita, contornando-a em sentido horário e assim sucessivamente até completar o número de crianças necessárias para a amostra.

Foram selecionados oito estudantes universitários e treinados pelos pesquisadores res- 
ponsáveis pela condução da investigação para a abordagem familiar, aplicação dos questionários, utilização da balança digital e do estadiômetro portátil. Para cada dupla de pesquisadores de campo foi entregue o material de trabalho composto por: mochila, lápis, caneta, prancheta, estadiômetro portátil, balança digital, cópia do mapa do setor censitário e questionários numerados sequenciados impressos. Cada final de tarde os questionários eram recolhidos pelos responsáveis pela pesquisa para revisão e feedback sobre as possíveis inconsistências aos pesquisadores de campo.

\section{Variáveis e fontes de dados}

Os questionários continham três módulos: o primeiro com as questões sobre sibilância do questionário resumido, modificado e traduzido para o português do EISL 2,11,12. Apesar de o questionário utilizado estar validado para as crianças de até 3 anos de idade 2, optou-se por estender a aplicação aos menores de 5 anos em razão do impacto que este grupo representa nas hospitalizações por asma. O segundo módulo constou de questões sobre a identificação das condições socioeconômicas, ambientais, gestação, parto e nascimento da criança; e o terceiro de questões sobre as condições de saúde.

O pré-teste do questionário foi realizado com uma amostra de crianças em um setor censitário de Cuiabá não participante da pesquisa. A questão sobre o uso de antileucotrienos foi excluída em razão da dificuldade de compreensão pelas famílias entrevistadas no teste piloto.

Foi calculado o alfa de Cronbach para os módulos de sibilância e condições de saúde, apresentando os seguintes valores, respectivamente: 0,83 e 0,79 . Com exceção da questão sobre o uso de antileucotrieno, não houve nenhuma outra que necessitasse ser retirada do questionário por gerar mudanças importantes na consistência interna.

A criança foi considerada como tendo sibilância se tivesse apresentado sibilos ou "chiado" no peito nos últimos 12 meses, com necessidade ou não do uso de broncodilatador e independentemente da frequência de sibilos.

Foi considerado como tendo "doença grave na vida" aquelas crianças cujas mães referiram sim à questão "Alguma vez na vida seu filho (...) esteve gravemente doente?”. Quando a mãe/responsável referia ter uma indústria a até três quadras da residência, em qualquer direção, foi considerado como "indústria próximo à residência".

O estado nutricional ao nascimento foi avaliado por meio do indicador "peso para idade" como registrado na caderneta da criança. Aque- las com peso ao nascer menor que $2.500 \mathrm{~g}$ foram classificadas como baixo peso e as com peso maior ou igual a $2.500 \mathrm{~g}$ como peso adequado ao nascimento.

Cada criança foi medida com antropômetro portátil com escala de precisão de 0,1cm (Sanny, São Paulo, Brasil) e pesada com balança digital da marca BIC, modelo Equilibrium (Bicmed, São Paulo, Brasil) com capacidade para até $180 \mathrm{~kg}$ e precisão de $100 \mathrm{~g}$, posicionada em superfície lisa e plana. O peso e estatura/altura foram tomados como medidas contínuas.

$\mathrm{O}$ peso atual para idade foi categorizado em baixo, adequado ou elevado, tomando-se por base os valores de referência da Organização Mundial da Saúde (OMS) 13, quais sejam escore $\mathrm{Z}$ de -2 a 2 peso adequado para a idade. Valores abaixo ou acima desses foram considerados baixo peso e peso elevado, respectivamente.

Verificou-se a completude ou não do esquema vacinal para a idade da criança, de acordo com o calendário básico do Ministério da Saúde, por meio do Cartão de Vacina ou Caderneta da Criança, que foi solicitado à mãe ou responsável no momento da entrevista.

Utilizou-se o instrumento CAGE (Cut-down; Annoyed; Guilty \& Eye-opener) para o rastreamento de alcoolismo entre as mães, o qual consiste de quatro questões que são inseridas entremeadas a outras questões de ordem diferenciadas ao longo do questionário utilizado. Considera-se CAGE positivo quando duas ou mais respostas são positivas para as questões do instrumento 14 .

Os dados foram digitados em dupla entrada no programa Epi Info 3.5.1 (Centers for Disease Control and Prevention, Atlanta, Estados Unidos) por profissionais diferentes, e analisados posteriormente quanto a inconsistências. Ao identificar quaisquer inconsistências, os formulários eram novamente revisados e realizava-se a correção no banco de dados. Foram excluídos seis questionários dos 739 em razão de inconsistência no registro dos dados.

\section{Análise de dados}

A variável dependente deste estudo é a presença de sibilância nos últimos 12 meses de vida. As variáveis independentes foram hierarquizadas nos níveis distal, intermediário e proximal.

O modelo teórico adotado foi baseado na proposta da OMS 15, que agrupa os fatores de risco em modificáveis e não modificáveis. Agregouse ainda esses fatores de acordo com os níveis hierarquizados, partindo do distal para o proximal (Figura 1).

No nível distal foram incluídas aquelas variáveis relacionadas às condições socioeconômicas: 


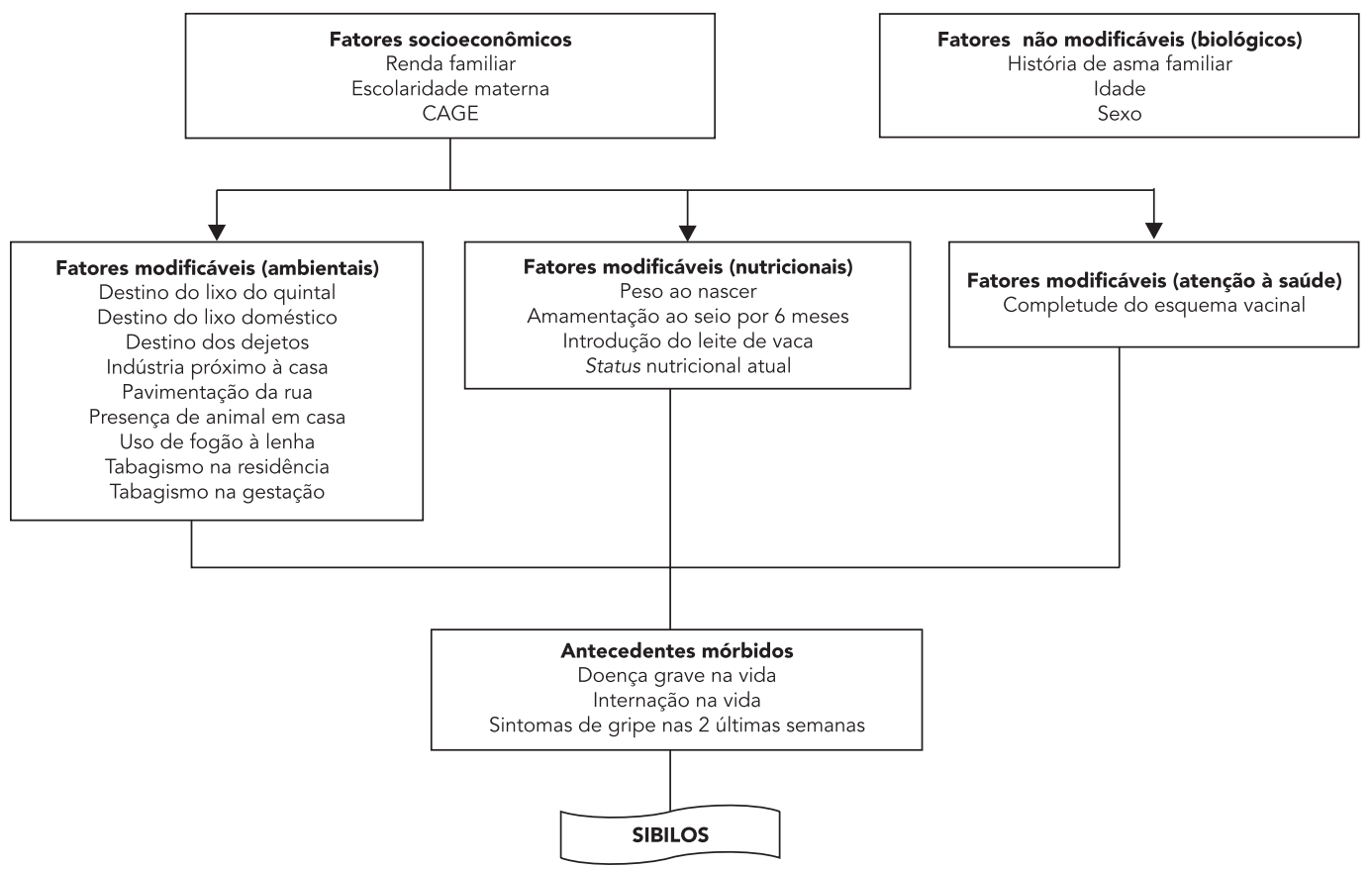

renda familiar em salários mínimos (correspondente a R\$ 510,00 à época), escolaridade materna e CAGE. Optou-se pelo ponto de corte da renda familiar em dois salários mínimos por corresponder aproximadamente aos rendimentos medianos das famílias da amostra. Incluiu-se ainda nesse nível os fatores não modificáveis como o sexo, idade e histórico familiar de asma.

No nível intermediário, as variáveis foram incluídas em três categorias de análise: fatores modificáveis ambientais, fatores modificáveis nutricionais e fatores modificáveis relacionados à atenção à saúde. São fatores modificáveis ambientais o destino do lixo doméstico e do quintal, destino dos dejetos, presença de indústria próximo à casa, pavimentação da rua, possuir animal de estimação em casa, uso de fogão à lenha, tabagismo na residência e tabagismo na gestação. Os fatores modificáveis nutricionais incluem o peso ao nascer, amamentação ao seio por seis meses ou mais, introdução de leite de vaca em qualquer momento da vida, status nutricional atual. Quanto aos fatores modificáveis relacionados à atenção à saúde, foi incluída a completude ou não do esquema vacinal.
No nível proximal foram incluídos os antecedentes mórbidos, quais sejam: doença grave e internação alguma vez na vida, sintomas de gripe nas últimas duas semanas.

Procedeu-se à análise bivariada e cálculo de odds ratio (OR) bruta com intervalo de 95\% de confiança (IC95\%) para avaliação das possíveis associações entre as variáveis estudadas e o desfecho.

$\mathrm{Na}$ análise de regressão logística múltipla foram incluídas aquelas variáveis que atingiram um nível de significância de $20 \%$ na bivariada. Considerando o nível distal como determinante dos demais, as variáveis desse nível foram analisadas separadamente e, aquelas estatisticamente significantes $(\mathrm{p}<0,05)$, integraram os ajustes das variáveis dos níveis subsequentes. Esse procedimento foi repetido sucessivamente até o nível proximal.

Por ser uma amostra complexa, as análises foram expandidas, considerando-se os pesos e o plano amostral, tendo por base a população segundo dados do Censo Demográfico de 2000 (IBGE. http://www.ibge.gov.br), disponível à época. 
As análises foram realizadas com o auxílio da biblioteca survey versão 3.29, do R versão 2.15.1 (The R Foundation for Statistical Computing, Viena, Áustria; http://www.r-project.org).

\section{Considerações éticas}

O estudo foi aprovado pelo Comitê de Ética em Pesquisa do Hospital Universitário Júlio Muller (HUJM) da Fundação Universidade Federal de Mato Grosso, em 09 de junho de 2010 (protocolo 770/CEP-HUJM/2010). Todos os responsáveis pelas crianças foram orientados sobre os objetivos da pesquisa e foram incluídos aqueles cujas assinaturas constaram no Termo de Consentimento Livre e Esclarecido.

\section{Resultados}

Foram entrevistadas 733 crianças. Suas famílias residem na maioria das vezes em casas/apartamentos próprios $(64,5 \%)$, de alvenaria (94\%), com água encanada $(94,8 \%)$, os dejetos são destinados em fossas sépticas ou esgoto $(94,4 \%)$ e moram em ruas com asfaltamento (59,5\%). Contudo, uma parcela ainda lança os dejetos a céu aberto $(4,4 \%)$, assim como o lixo doméstico (5,3\%). Em caso de doença, $80,3 \%$ utilizam predominantemente o serviço público de saúde e 19,7\% apenas o serviço privado.

A renda média das famílias é de $\mathrm{R} \$ 1.718,85$ com mediana de $\mathrm{R} \$ 1.000,00$, ou seja, metade das famílias apresentava renda média menor do que três salários mínimos à época; com renda média per capita de $\mathrm{R} \$ 393,08$ e mediana de $\mathrm{R} \$ 200,00$, que equivale a menos de 1 dólar por dia/pessoa. A idade média das mães é de 27,8 anos (IC95\%: 26,9-28,6), a maioria estudou até o ensino médio incompleto (51\%), reside no Município de Cuiabá há aproximadamente 20 anos e no mesmo endereço por oito anos.

Dentre as crianças analisadas, $52,6 \%$ eram do sexo masculino e $47,4 \%$ do feminino, $22,7 \%$ menores de 1 ano e $77,3 \%$ de 1 a 4 anos. Tanto em relação ao sexo quanto à faixa etária, o padrão da amostra é similar à distribuição da população menor de 5 anos de idade no Município de Cuiabá segundo dados do IBGE (Cidades@. http:// www.ibge.gov.br/cidadesat/topwindow.htm?1, acessado em 20/Out/2011).

As crianças foram amamentadas ao seio em média e mediana de cinco meses, com introdução de leite em pó (leite de vaca modificado) também a esta época e introdução do leite de vaca por volta dos 11 meses de vida; 10,8\% apresentaram algum atraso no esquema vacinal.
No conjunto das crianças menores de 5 anos, verificou-se que o primeiro episódio de sibilância ocorreu em média aos 9,4 meses de idade (IC95\%: 8,3-10,4), enquanto as menores de 1 ano sibilaram pela primeira vez aos 3,7 meses (IC95\%: 2,9-4,6). A prevalência de sibilância nos últimos 12 meses foi de 43,2\%, despertares noturnos frequentes por sintomas $15 \%$, consultas em serviço de emergência $41,3 \%$ e dificuldade respiratória $37,2 \%$. Entre as crianças de 1 ano de idade, a prevalência de sibilância foi de 52,1\%, reduzindo a partir daí até $38,1 \%$ aos 3 e $46,4 \%$ aos 4 anos. A prevalência de diagnóstico médico de asma, por outro lado, é menor no lo ano de vida $(9,6 \%)$, com pico aos 3 anos (14,6\%).

Como se observa na Tabela 1 , tanto a renda familiar menor que dois salários mínimos quanto a escolaridade materna e o rastreamento positivo para alcoolismo não se mostraram associados significativamente à presença de sibilos nos últimos 12 meses de vida. O histórico familiar de asma foi $98 \%$ mais frequente nas crianças sibilantes, de forma significativa $(\mathrm{OR}=1,98$; IC95\%: 1,05-3,73; $\mathrm{p}=0,041)$. A prevalência de sibilos foi semelhante nas crianças independentemente da classificação etária; e 34\% maior entre as crianças do sexo masculino (OR = 1,34; IC95\%: 1,02$1,77 ; \mathrm{p}=0,042)$.

$\mathrm{Na}$ Tabela 2, pode-se verificar que possuir fogão à lenha, o destino do lixo doméstico e o destino dos dejetos também não foram associados com sibilância; enquanto as crianças cujas famílias queimam/enterram o lixo do quintal apresentam chance $57 \%$ menor de apresentar sibilância do que aquelas onde há coleta pública disponível (OR = 0,43; IC95\%: 0,17-1,06; p = 0,075). Crianças cujas residências estão localizadas próximas a indústrias não apresentaram maior chance de sibilância $(\mathrm{OR}=1,20$; IC95\%: $0,74-1,95 ; p=0,460$ ). Aquelas que moravam em ruas não pavimentadas apresentaram chance $25 \%$ menor de sibilância nos últimos 12 meses, mas sem significância estatística $(\mathrm{OR}=0,75$; IC95\%: 0,55-1,01; $p=0,208)$. Também não foram associados à sibilância nos últimos 12 meses o fato da criança possuir animal doméstico, a mãe ter sido tabagista durante a gestação e ter tabagistas na residência.

Em relação às variáveis modificáveis nutricionais (Tabela 2), a não amamentação ao seio por no mínimo seis meses representou uma chance $66 \%$ maior de sibilância entre as crianças $(\mathrm{OR}=$ 1,66; IC95: 1,12-2,45; p = 0,015). A introdução do leite de vaca na dieta representou uma chance $58 \%$ maior de ter sibilos (OR $=1,58$; IC $95 \%$ : $1,01-2,45 ; \mathrm{p}=0,050)$. Tanto o peso ao nascer $(\mathrm{OR}=$ 1,53; IC95\%: 0,75-3,11; $\mathrm{p}=0,249$ ) quanto o peso para idade atual não se mostraram associados 
Distribuição dos casos de sibilância nos últimos 12 meses em menores de 5 anos de idade, odds ratio (OR) bruta e respectivos intervalos de 95\% de confiança (IC95\%) segundo variáveis do nível distal *. Cuiabá, Mato Grosso, Brasil, 2010.

\begin{tabular}{|c|c|c|c|c|c|c|}
\hline \multirow[t]{2}{*}{ Variável } & \multicolumn{2}{|c|}{ Sibilos (\%) ** } & \multirow[t]{2}{*}{ Total } & \multirow[t]{2}{*}{ OR $_{\text {bruta }}$} & \multirow{2}{*}{$\begin{array}{c}\text { IC95\% } \\
\left(\mathrm{OR}_{\text {bruta }}\right)\end{array}$} & \multirow{2}{*}{$\begin{array}{l}\text { Valor } \\
\text { de } p\end{array}$} \\
\hline & Sim & Não & & & & \\
\hline \multicolumn{7}{|c|}{ Fatores socioeconômicos } \\
\hline \multicolumn{7}{|c|}{ Renda familiar (salários mínimos) } \\
\hline$<2$ & 54,1 & 54,1 & 54,1 & 0,99 & $0,65-1,53$ & 0,989 \\
\hline$\geq 2$ & 45,9 & 45,9 & 45,9 & 1,00 & & \\
\hline \multicolumn{7}{|c|}{ Escolaridade materna (anos) } \\
\hline$\leq 8$ & 48,7 & 52,8 & 51,0 & 0,84 & $0,54-1,31$ & 0,470 \\
\hline$>8$ & 51,3 & 47,2 & 49,0 & 1,00 & & \\
\hline \multicolumn{7}{|l|}{ CAGE positivo } \\
\hline Sim & 3,9 & 2,5 & 3,1 & 1,58 & $0,62-4,06$ & 0,342 \\
\hline Não & 96,1 & 97,5 & 96,9 & 1,00 & & \\
\hline \multicolumn{7}{|c|}{ Fatores não modificáveis (biológicos) } \\
\hline \multicolumn{7}{|c|}{ História familiar de asma } \\
\hline Sim & 13,1 & 7,1 & 9,7 & 1,98 & $1,05-3,73$ & 0,041 \\
\hline Não & 86,9 & 92,9 & 90,3 & 1,00 & & \\
\hline \multicolumn{7}{|c|}{ Classificação etária (anos) } \\
\hline$<1$ & 41,9 & 44,5 & 43,3 & 0,89 & $0,50-1,56$ & 0,689 \\
\hline $1-3$ & 40,8 & 40,3 & 40,5 & 0,83 & $0,48-1,42$ & 0,499 \\
\hline$\geq 4$ & 17,3 & 15,2 & 16,1 & 1,00 & & \\
\hline \multicolumn{7}{|l|}{ Sexo } \\
\hline Masculino & 56,8 & 49,5 & 52,6 & 1,34 & $1,02-1,77$ & 0,042 \\
\hline Feminino & 43,2 & 50,5 & 47,4 & 1,00 & & \\
\hline
\end{tabular}

* Os valores de OR, IC95\% e valor de p foram calculados considerando os pesos e o plano amostral;

$\star \star$ Valores relativos (\%).

significativamente à sibilância. A variável relacionada à atenção à saúde, a incompletude do esquema vacinal, não esteve associada à sibilância.

As crianças sibilantes foram mais frequentemente classificadas como tendo uma doença grave alguma vez na vida (53\%) $(\mathrm{OR}=1,53$; IC95\%: 1,09-2,15; $\mathrm{p}=0,018$ ) e mais frequentemente internadas do que as outras $(61 \%)(\mathrm{OR}=$ 1,61; IC95\% 1,10-2,36; $p=0,019)$. Essas crianças também apresentaram maior frequência de sintomas de gripe nas duas últimas semanas (57\%) (OR = 1,57; IC95\%: 1,04-2,35; $\mathrm{p}=0,035)$, como se observa na Tabela 3.

Na análise de regressão logística múltipla (Tabela 4), os fatores socioeconômicos não atingiram significância para inclusão no modelo. Após ajustes pelas variáveis do nível distal ao proximal, permaneceram associados à sibilância nos últimos 12 meses a não amamentação ao seio por no mínimo seis meses, a história familiar de asma, o sexo masculino e a história de doença prévia. Crianças não amamentadas ao seio por seis meses ou mais apresentaram chance $91 \%$ maior de sibilância, aquelas com histórico familiar de asma tiveram duas vezes mais chance do desfecho e as do sexo masculino $50 \%$ maior chance. A existência de doença grave alguma vez na vida representou chance $81 \%$ maior de a criança apresentar sibilância.

\section{Discussão}

Comparar a prevalência de sibilância na literatura é um desafio em razão dos critérios para definição de caso, bem como das diferentes faixas etárias analisadas nos estudos publicados. No entanto, a prevalência de sibilância e de diagnóstico médico de asma no presente estudo está situada entre os valores mais altos considerando os limites de variação descritos na literatura nacional 2,3,10,17,18.

Tanto os fatores modificáveis como aqueles não modificáveis estiveram associados à 
Tabela 2

Distribuição dos casos de sibilância nos últimos 12 meses em menores de 5 anos de idade, odds ratio (OR) bruta e respectivos intervalos de $95 \%$ de confiança (IC95\%) segundo variáveis do nível intermediário *. Cuiabá, Mato Grosso, Brasil, 2010.

\begin{tabular}{|c|c|c|c|c|c|c|}
\hline \multirow[t]{2}{*}{ Variável } & \multicolumn{2}{|c|}{ Sibilos (\%) ** } & \multirow[t]{2}{*}{ Total } & \multirow[t]{2}{*}{ OR $_{\text {bruta }}$} & \multirow{2}{*}{$\begin{array}{c}\text { IC95\% } \\
\left(O_{\text {bruta }}\right)\end{array}$} & \multirow[t]{2}{*}{ Valor de $p$} \\
\hline & Sim & Não & & & & \\
\hline \multicolumn{7}{|l|}{ Possui fogão à lenha } \\
\hline $\operatorname{Sim}$ & 6,3 & 8,5 & 7,5 & 0,72 & $0,37-1,40$ & 0,343 \\
\hline Não & 93,7 & 91,5 & 92,5 & 1,00 & & \\
\hline \multicolumn{7}{|l|}{ Destino do lixo doméstico } \\
\hline Queima/Enterra & 1,5 & 4,1 & 3,0 & 0,37 & $0,11-1,24$ & 0,116 \\
\hline Coleta pública & 98,5 & 95,9 & 97,0 & 1,00 & & \\
\hline \multicolumn{7}{|l|}{ Destino do lixo do quintal } \\
\hline Queima/Enterra & 3,2 & 7,1 & 5,4 & 0,43 & $0,17-1,06$ & 0,075 \\
\hline Coleta pública & 96,8 & 92,9 & 94,6 & 1,00 & & \\
\hline Céu aberto & 4,1 & 4,7 & 4,5 & 0,87 & $0,42-1,82$ & 0,726 \\
\hline Esgoto/Fossa séptica & 95,9 & 95,3 & 95,5 & 1,00 & & \\
\hline \multicolumn{7}{|c|}{ Presença de indústria próxima à casa } \\
\hline $\operatorname{Sim}$ & 29,7 & 26,0 & 27,7 & 1,20 & $0,74-1,95$ & 0,460 \\
\hline Não & 70,3 & 74,0 & 72,4 & 1,00 & & \\
\hline \multicolumn{7}{|c|}{ Pavimentação da rua da casa } \\
\hline Não & 36,5 & 43,5 & 40,5 & 0,75 & $0,55-1,01$ & 0,208 \\
\hline Sim & 63,5 & 56,5 & 59,5 & 1,00 & & \\
\hline \multicolumn{7}{|l|}{ Possui animal em casa } \\
\hline Sim & 58,6 & 53,5 & 55,7 & 1,23 & $0,83-1,82$ & 0,302 \\
\hline Não & 41,4 & 46,5 & 44,3 & 1,00 & & \\
\hline \multicolumn{7}{|c|}{ Tabagismo durante gravidez } \\
\hline $\operatorname{Sim}$ & 9,8 & 11,4 & 10,7 & 0,84 & $0,47-1,52$ & 0,577 \\
\hline \multicolumn{7}{|l|}{ Tabagismo na residência } \\
\hline Sim & 34,8 & 33,8 & 34,2 & 1,04 & $0,76-1,42$ & 0,776 \\
\hline Não & 65,2 & 66,2 & 65,8 & 1,00 & & \\
\hline \multicolumn{7}{|c|}{ Variáveis modificáveis (nutricionais) } \\
\hline \multicolumn{7}{|c|}{ Amamentação ao seio por 6 meses ou mais } \\
\hline Não & 88,7 & 82,5 & 85,2 & 1,66 & $1,12-2,45$ & 0,015 \\
\hline Sim & 11,3 & 17,5 & 14,8 & 1,00 & & \\
\hline \multicolumn{7}{|c|}{ Introdução do leite de vaca } \\
\hline $\operatorname{Sim}$ & 68,8 & 58,2 & 62,8 & 1,58 & $1,01-2,45$ & 0,050 \\
\hline Não & 31,2 & 41,8 & 37,2 & 1,00 & & \\
\hline \multicolumn{7}{|l|}{ Peso ao nascer (gramas) } \\
\hline$<2.500$ & 9,4 & 6,3 & 7,7 & 1,53 & $0,75-3,11$ & 0,249 \\
\hline$\geq 2.500$ & 90,6 & 93,7 & 92,3 & 1,00 & & \\
\hline \multicolumn{7}{|l|}{ Peso para idade atual } \\
\hline Baixo peso & 0,5 & 1,3 & 0,9 & 2,59 & $0,23-28,45$ & 0,442 \\
\hline Peso adequado & 97,7 & 96,9 & 97,3 & 1,00 & & \\
\hline Sobrepeso/Obeso & 1,8 & 1,8 & 1,8 & 2,58 & $0,16-40,86$ & 0,506 \\
\hline \multicolumn{7}{|c|}{ Variáveis modificáveis (atenção à saúde) } \\
\hline Incompletude do esquer & & & & & & \\
\hline Sim & 13,2 & 9,4 & 11,0 & 1,47 & $0,86-2,50$ & 0,167 \\
\hline Não & 86,8 & 90,6 & 89,0 & 1,00 & & \\
\hline
\end{tabular}

* Os valores de OR, IC95\% e valor de p foram calculados considerando os pesos e o plano amostral;

** Valores relativos (\%). 
Distribuição dos casos de sibilância nos últimos 12 meses em menores de 5 anos de idade, odds ratio (OR) bruta e respectivos intervalos de 95\% de confiança (IC95\%) segundo variáveis do nível proximal *. Cuiabá, Mato Grosso, Brasil, 2010.

\begin{tabular}{|c|c|c|c|c|c|c|}
\hline \multirow[t]{2}{*}{ Variável } & \multicolumn{2}{|c|}{ Sibilos $(\%) * \star$} & \multirow[t]{2}{*}{ Total } & \multirow[t]{2}{*}{ OR $_{\text {bruta }}$} & \multirow[t]{2}{*}{ IC95\% } & \multirow{2}{*}{$\begin{array}{l}\text { Valor } \\
\text { de } p\end{array}$} \\
\hline & $\operatorname{Sim}$ & Não & & & & \\
\hline \multicolumn{7}{|c|}{ Fatores modificáveis (antecedentes mórbidos) } \\
\hline \multicolumn{7}{|c|}{ Doença grave na vida } \\
\hline $\operatorname{Sim}$ & 42,4 & 32,4 & 36,7 & 1,53 & $1,09-2,15$ & 0,018 \\
\hline Não & 57,6 & 67,6 & 63,3 & 1,00 & & \\
\hline \multicolumn{7}{|c|}{ Internação na vida (qualquer motivo) } \\
\hline Sim & 36,1 & 25,9 & 30,3 & 1,61 & $1,10-2,36$ & 0,019 \\
\hline Não & 63,9 & 74,1 & 69,7 & 1,00 & & \\
\hline \multicolumn{7}{|c|}{ Sintomas de gripe nas últimas 2 semanas } \\
\hline $\operatorname{Sim}$ & 64,3 & 53,4 & 58,1 & 1,57 & $1,04-2,35$ & 0,035 \\
\hline Não & 35,7 & 46,6 & 41,9 & 1,00 & & \\
\hline
\end{tabular}

* Os valores de OR, IC95\% e p-valor foram calculados considerando os pesos e o plano amostral;

** Valores relativos (\%).

sibilância. Esses fatores podem estar relacionados tanto à provável atopia quanto a infecções. Dentre as doenças presentes na infância, as principais são as infecções respiratórias e estas se configuram como fator de risco para asma $10,17,18$, com destaque para o papel das infecções virais, que podem causar sibilância em graus variados 19,20,21. As mães podem superestimar a gravidade da doença do filho, no entanto, é pouco provável que diga que o mesmo está doente quando de fato não está.

Quanto ao histórico familiar de asma (mães, pais ou irmãos), este é reconhecidamente um dos mais importantes determinantes de asma, principal doença crônica que cursa com sibilância na infância $6,7,22,23$. O sexo masculino também é um fator de risco conhecido para asma 6 , apesar de não configurar fator de risco para todos os fenótipos de sibilância; tendo sido associado ao fenótipo sibilante persistente, mas não ao fenótipo transitório 24. Tanto o histórico familiar de asma quanto o sexo masculino são considerados critérios definidores de caso de asma em crianças com sibilância recorrente 22. É provável que uma parcela de casos da doença esteja subdiagnosticada entre as crianças em Cuiabá.

A amamentação ao seio por tempo menor do que seis meses foi associada à sibilância entre as crianças em Cuiabá. Sabe-se que o aleitamento materno é o aconselhado, mesmo sabendo que a relação entre este e o desenvolvimento de asma ainda é controverso, devido à complexidade da interação entre os alimentos em geral e o desenvolvimento do sistema imune da criança 25 , e também da dieta da mãe durante a gestação 26 . A relação entre amamentação exclusiva e sibilância é controversa, diferindo entre o nível de desenvolvimento dos países, o diagnóstico ou não de asma e atopia, e o ponto de corte utilizado para o tempo de aleitamento materno exclusivo 27,28,29,30.

Estudos têm demonstrado haver evidências de relação causal entre tabagismo passivo e deflagração de casos de sibilância 31, entretanto resultados em que não há associação têm sido relatados 32 . Responsáveis por crianças com doenças respiratórias têm a tendência a subnotificar o tabagismo 33, o que também poderia justificar os achados de não associação verificados no presente estudo.

Nesta pesquisa, a renda não está associada à sibilância em crianças. Os estudos mostram-se contraditórios em relação à renda e associação com sibilância ou asma, com registro tanto de ausência de associação 34,35 quanto de associação positiva 36 .

Optou-se por analisar como desfecho a presença de sibilância nos 12 últimos meses, ainda que diferentes fenótipos de sibilância estejam envolvidos neste desfecho, com fatores de risco também diferentes para cada fenótipo 24 .

Episódios de sibilância diminuem em frequência com o aumento da idade, enquanto o diagnóstico de asma aumenta com a idade, com picos aos 3-4 anos 37. Também no caso do presente estudo foi observada essa relação, com maior prevalência dos episódios de sibilância entre as crianças de 1 ano de idade e redução a 
Distribuição dos casos de sibilância nos últimos 12 meses em menores de 5 anos de idade, odds ratio (OR) ajustada e respectivos intervalos de 95\% de confiança (IC95\%) segundo variáveis do nível distal ao proximal *. Cuiabá, Mato Grosso, Brasil, 2010.

\begin{tabular}{|c|c|c|c|}
\hline Variável & OR $_{\text {ajustada }}$ & IC95\% (OR $\left.{ }_{\text {ajustada }}\right)$ & Valor de $p$ \\
\hline \multicolumn{4}{|l|}{ Nível distal (biológicos) ** } \\
\hline \multicolumn{4}{|l|}{ História de asma familiar } \\
\hline Sim & 2,02 & $1,06-3,87$ & 0,040 \\
\hline \multicolumn{4}{|l|}{ Sexo } \\
\hline Masculino & 1,50 & $1,07-2,11$ & 0,024 \\
\hline \multicolumn{4}{|c|}{ 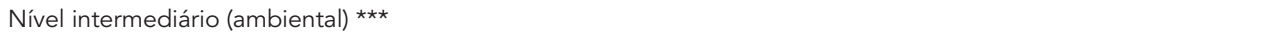 } \\
\hline \multicolumn{4}{|c|}{ Destino do lixo doméstico } \\
\hline Queima ou enterra & 1,17 & $0,15-9,20$ & 0,885 \\
\hline \multicolumn{4}{|c|}{ Destino do lixo do quintal } \\
\hline Queima ou enterra & 0,38 & $0,11-1,31$ & 0,136 \\
\hline \multicolumn{4}{|l|}{ Pavimentação da rua } \\
\hline Não & 0,80 & $0,60-1,06$ & 0,136 \\
\hline \multicolumn{4}{|c|}{ Nível intermediário (nutricional) \# } \\
\hline \multicolumn{4}{|c|}{ Amamentação ao seio por 6 meses } \\
\hline Não & 1,91 & $1,18-3,06$ & 0,012 \\
\hline \multicolumn{4}{|c|}{ Introdução do leite de vaca } \\
\hline Sim & 1,40 & $0,88-2,24$ & 0,170 \\
\hline \multicolumn{4}{|c|}{ Nível intermediário (atenção à saúde) \#\# } \\
\hline \multicolumn{4}{|c|}{ Completude do esquema vicinal } \\
\hline Não & 1,29 & $0,79-2,11$ & 0,321 \\
\hline \multicolumn{4}{|c|}{ Nível proximal (antecedentes mórbidos) \#\#\# } \\
\hline \multicolumn{4}{|l|}{ Doença prévia } \\
\hline Sim & 1,81 & $1,05-3,14$ & 0,042 \\
\hline \multicolumn{4}{|l|}{ Internação na vida } \\
\hline Sim & 0,74 & $0,42-1,33$ & 0,323 \\
\hline \multicolumn{4}{|c|}{ Sintoma de gripe nas 2 últimas semanas } \\
\hline Sim & 1,34 & $0,89-2,01$ & 0,167 \\
\hline
\end{tabular}

* As variáveis socioeconômicas do nível distal não atingiram significância para inclusão no modelo final;

** Ajustado pelas variáveis do nível distal - não modificáveis (biológicos);

*** Ajustado pelas variáveis do nível distal (biológicos) e pelas variáveis do nível intermediário (ambiental);

\# Ajustado pelas variáveis do nível distal (biológicos) e pelas variáveis do nível intermediário (nutricional);

\#\# Ajustado pelas variáveis do nível distal (biológicos), pela variável "amamentação ao seio por 6 meses" e pela variável

"completude do esquema vacinal";

\#\#\# Ajustado pelas variáveis do nível distal (biológicos), pela variável "amamentação ao seio por 6 meses" e pelas variáveis do nível proximal (antecedentes mórbidos).

partir daí; enquanto os casos de diagnóstico médico da asma aumentaram com a idade. A diminuição dos casos de sibilância com a idade pode estar relacionada à redução dos casos de doenças respiratórias virais, mais acentuados nos primeiros anos de vida. Em relação ao diagnóstico de asma, a probabilidade aumenta com a idade em razão da maior facilidade do diagnóstico médico nas crianças mais velhas e da experiência com repetidas crises de sibilância.
Como limitação deste estudo pode-se citar que as crianças não foram examinadas por médico para identificação da sibilância, tendo sido considerado apenas o relato da mãe/responsável. Não obstante, este trabalho não se propunha à realização de exame clínico, e o uso de questionários padronizados constitui um método não invasivo seguro para identificação de crianças sibilantes, bem como daquelas com sibilância recorrente, que caracterizam o grupo de maior ris- 
co para o desenvolvimento de asma. Além disso, o questionário utilizado é validado para crianças de até 36 meses de vida. Entretanto, é provável que não haja diferenças importantes em relação às respostas da mãe ou responsável das crianças de 4 anos em relação àquelas de 3 anos. As diferenças encontradas podem ser próprias das variações de idade.

Episódios menores de sibilos podem não ser identificados pelas mesmas e os entrevistados podem não se lembrar de todos os fatos que ocorreram ao longo de um ano, ou ainda, a data do primeiro episódio pode não ser muito precisa especialmente em razão de ter sido estendida a aplicação do questionário aos menores de 5 anos. No entanto, considera-se que ao analisar esse grupo o efeito da memória sobre as respostas seja reduzido se comparado a crianças mais velhas. Outro aspecto diz respeito à classificação do estado nutricional ao nascimento, que não leva em conta as diferenças de peso próprias da idade gestacional ao nascer, com consequentes implicações para a saúde da criança. Esse, porém, é um critério amplamente utilizado, recomendado pela Rede Interagencial de Informações para a Saúde (RIPSA) e adotado pelo Ministério da Saúde 38.

Em relação aos estudos de prevalência, pode-se citar que as OR são superestimadas em relação às razões de prevalências, bem como podem apresentar intervalos de confiança mais amplos tanto para a OR bruta quanto para a ajustada 39. Entretanto, a OR possibilita a identificação de possíveis associações nas quais a razão de prevalência pode levar a falsas conclusões ${ }^{40}$. Estudos comparativos entre as duas medidas utilizando-se técnica de regressão lo- gística resultam em modelos finais semelhantes, tanto em situações de alta quanto de baixa prevalência do desfecho 39 .

A abordagem hierarquizada realizada no presente estudo possibilita quantificar a contribuição de cada nível hierárquico na determinação do risco. Por ser uma amostra complexa, ao incorporar, na análise, os pesos e a expansão amostral foi possível tornar a análise ainda mais robusta.

Em localidades com diferentes características e padrões de desenvolvimento pode haver variação na proporção de asma relacionada à atopia 41 , com predominância de atopia em crianças asmáticas em um centro desenvolvido 42 . Enquanto em relação à sibilância, houve maior prevalência de sibilantes não atópicos em um centro em desenvolvimento 43 .

Identificar qual a influência dos fatores modificáveis e não modificáveis, bem como das infecções e da atopia na determinação da elevada prevalência de sibilância na infância em Cuiabá é um desafio para futuros estudos, em especial para diferenciar aquelas crianças com maior risco para desenvolver asma persistente. Isso é relevante, pois as taxas de internação por asma em menores de 15 anos no Estado de Mato Grosso estão entre as mais altas da Região Amazônica 44, e Cuiabá apresenta alta prevalência de atendimentos ambulatoriais por asma em menores de 5 anos 45 .

Conclui-se que a prevalência de sibilância em crianças menores de 5 anos de idade em Cuiabá é elevada e está associada ao histórico familiar de asma, sexo masculino, amamentação ao seio menor do que seis meses e presença de doença prévia na vida. 


\section{Resumen}

La prevalencia de sibilancias en Brasil es alta, variando entre las distintas regiones del país y con factores de riesgo diferentes. Con el objetivo de analizar la prevalencia y los factores asociados con sibilancias en niños menores de 5 años en Cuiabá, Mato Grosso, Brasil, se llevó a cabo un estudio transversal, cuya muestra estuvo constituida por 733 niños. Utilizamos un instrumento estandarizado, resumido del Estudio Internacional de Sibilancias en Lactantes (EISL), con el fin de analizar los factores asociados con sibilancias se aplicó la regresión logística con un enfoque jerárquico. La prevalencia de sibilancias en los últimos 12 meses fue un 43,2\%. Se asocia con las sibilancias: no lactancia durante un periodo de 6 meses o más (OR ajustada =1,91; IC95\%: 1,18-3,06), el diagnóstico de asma en familia (OR ajustada = 2,02; IC95\%: 1,06-3,87), enfermedad previa (OR ajustada = 1,81; IC95\%: 1,05-3,14) y el sexo masculino (OR ajustada = 1,50; IC95\%: 1,07-2,11). Se concluye que la prevalencia de sibilancias en los niños de esta edad en Cuiabá es alta y se asocia a factores relacionados con el asma, enfermedad previa y sexo masculino.

Sibilancia; Asma; Niño

\section{Referências}

1. Chong Neto HJ, Rosário NA, Solé D, Mallol J. Prevalência de sibilância recorrente em lactentes. J Pediatr (Rio J.) 2007; 83:357-62.

2. Bianca ACCD, Myagi K, Camargo L, Cezarin D, Wandalsen GF, Solé D. Estudo Internacional de sibilância em lactentes (EISL): validação de questionário escrito para lactentes com até 36 meses de vida na cidade de São Paulo. Rev Bras Alergia Imunopatol 2007; 30:232-9.

3. Bianca ACCD, Wandalsen GF, Mallol J, Solé D. Prevalência e gravidade da sibilância no primeiro ano de vida. J Bras Pneumol 2010; 36:402-9.

4. Alvim CG, Nunes S, Fernandes S, Camargos P, Fontes MJ. Corticoide oral e inalatório para tratamento de sibilância no primeiro ano de vida. J Pediatr (Rio J.) 2011; 87:314-8.

5. Neville RG, McCowan C, Hoskins G, Thomas G. Cross-sectional observations on the natural history of asthma. Br J Gen Practice 2001; 51:361-5.

\section{Colaboradores}

A. M. Rosa e E. Ignotti participaram do desenho do estudo, coleta e análise dos dados, discussão e revisão final do artigo. L. S. V. Jacobson colaborou na análise estatística e revisão final do artigo. C. Botelho contribuiu na discussão e revisão final do artigo

\section{Agradecimentos}

Os autores agradecem a Sílvia Líllian Rosinha Queiroz pelo imenso apoio na logística da pesquisa durante a fase de coleta de dados, e aos acadêmicos Aparecido Silva Santos, Danilo Vitor Camargo Prates, Denyth Sempio Justino, Fanni Cristina Rodrigues dos Santos, Jéssica Alves Oliveira, Luciney dos Santos Lara, Míria Brandão de Araújo e Pãmela Rodrigues da Silva pela participação na etapa de coleta de dados. Ao CNPq - CTSAÚDE/edital MCT/CNPq/CT-Saúde no 54/2009 - Doenças Respiratórias na Infância, processo o 557333/2009-1.
6. Global Initiative for Asthma. Global strategy for asthma management and prevention, 2010. http:// www.ginasthma.org/pdf/GINA_Report_2010.pdf (acessado em 20/Mar/2012)

7. Sociedade Brasileira de Pneumologia e Tisiologia. Diretrizes da Sociedade Brasileira de Pneumologia e Tisiologia para o manejo da asma: 2012. J Bras Pneumol 2012; 38 Suppl 1:S1-46.

8. Pearce N, Douwes J, Beasley R. How much asthma is really attributable to atopy? Thorax 1999; 54:268-72.

9. Cunha SS, Barreto ML, Fiaccone RL, Cooper PJ, Alcantara-Neves NM, Simões SM, et al. Asthma cases in childhood attributed to atopy in tropical area in Brazil. Rev Panam Salud Pública 2010; 28:405-11.

10. Barreto ML, Cunha SS, Fiaccone R, Esquivel R, Amorim LD, Alvim S, et al. Poverty, dirt, infections and non-atopic wheezing in children from a Brazilian urban center. Respir Res 2010; 11:167. 
11. Chong Neto HJ, Rosário NA. Expanding the application of a standardized questionnaire on recurrent wheezing in infancy. J Pediatr (Rio J.) 2009; 85:170-4.

12. Mallol J, García-Marcos L. Estudio Internacional de Sibilâncias en Lactantes (EISL). http://respirar. org/pdf/eisl/eisl_visiondeconjunto.pdf (acessado em 20/Jul/2012)

13. WHO Multicentre Growth Reference Study Group. WHO child growth standards based on length/ height, weight and age. Acta Paediatr Suppl 2006; 450:76-85.

14. Capriglione MJ, Monteiro MG, Masur J. Aplicação do questionário CAGE para detecção precoce da síndrome de dependência do álcool em 700 adultos na cidade de São Paulo. Rev Assoc Bras Psiquiatr 1985; 7:50-3.

15. World Health Organization. Preventing chronic diseases: a vital investment. Geneva: World Health Organization; 2005.

16. Wehrmeister FC, Peres KGA. Desigualdades regionais na prevalência de diagnóstico de asma em crianças: uma análise da Pesquisa Nacional por Amostra de Domicílios, 2003. Cad Saúde Pública 2010; 26:1839-52.

17. Medeiros D, Silva AR, Rizzo JA, Sarinho E, Mallol J, Solé D. Prevalência de sibilância e fatores de risco associados em crianças no primeiro ano de vida, residentes no Município de Recife, Pernambuco, Brasil. Cad Saúde Pública 2011; 27:1551-9.

18. Muiño A, Menezes AMB, Reichert FF, Duquia RP, Chatkin M. Padrões de sibilância respiratória do nascimento até o início da adolescência: coorte de Pelotas (RS) Brasil, 1993-2004. J Bras Pneumol 2008; 34:347-55.

19. Center for Disease Control and Prevention. Respiratory syncytial virus infection (RSV): infection and incidence. http://www.cdc.gov/rsv/about/in fection.html (acessado em 20/Jun/2012).

20. Nair H, Nokes DJ, Gessnere BD, Dheranif M, Madhi SA, Singleton RJ, et al. Global burden of acute lower respiratory infections due to respiratory syncytial virus in young children: a systematic review and meta-analysis. Lancet 2010; 375:1545-55.

21. Jackson DJ, Lemanske Jr. RF. The role of respiratory virus infections in childhood asthma inception. Immunol Allergy Clin North Am 2010; 30:513-22.

22. Castro-Rodrigues JA, Holberg CJ, Wright AL, Martinez FD. A clinical index to define risk of asthma in young children with recurrent wheezing. Am J Respir Crit Care Med 2000; 162:1403-6.

23. Martel MJ, Rey E, Malo JL, Perreault S, Beauchesne MF, Forget A, et al. Determinants of the incidence of childhood asthma: a two-stage case-control study. Am J Epidemiol 2009; 169:195-205.

24. Suh DI, Koh YY. Early childhood wheezing: various natural courses and their relationship to later asthma. Korean J Pediatr 2012; 55:259-64.

25. Schneider AP, Stein RT, Fritscher CC. O papel do aleitamento materno, da dieta e do estado nutricional no desenvolvimento de asma e atopia. J Bras Pneumol 2007; 33:454-62.

26. Devereux G. Meeting of the nutrition society. In: Proceedings of the Symposium on "Dietary Management of Disease”. London: Nutrition Society; 2010. p. 1-10.
27. Fredriksson P, Jaakkola N, Jaakkola JJK. Breastfeeding and childhood asthma: a six-year populationbased cohort study. BMC Pediatrics 2007; 7:39.

28. Ehlayel MS, Bener A. Duration of breast-feeding and the risk of childhood allergic diseases in a developing country. Allergy Asthma Proc 2008; 29:386-91.

29. Midodzi WK, Rowe BH, Majaesic CM, Saunders LD, Senthilselvan A. Early life factors associated with incidence of physician-diagnosed asthma in preschool children: results from the Canadian Early Childhood Development cohort study. J Asthma 2010; 47:7-13.

30. Björkstén B, Aït-Khaled N, Innes Asher M, Clayton TO, Robertson C. Global analysis of breastfeeding and risk of symptoms of asthma, rhinoconjunctivitis and eczema in 6-7 year old children: ISAAC Phase Three. Allergol Immunopathol 2011; 39: 318-25.

31. Centers for Disease Control and Prevention. The health consequences of involuntary exposure to tobacco smoke: a report of the Surgeon General. http://www.surgeongeneral.gov/library/reports/ secondhandsmoke/ (acessado em 20/Jul/2012).

32. Nguyen T, Lurie M, Gomez M, Reddy A, Pandya K, Medvesky M. The National Asthma Survey - New York State: association of the home environment with current asthma status. Public Health Rep 2010; 125:877-87.

33. Girardi L. Efeito da exposição a fumo passivo em lactentes hospitalizados com bronquiolite viral aguda no primeiro ano de vida [Dissertação de Mestrado]. Porto Alegre: Universidade Federal do Rio Grande do Sul; 2003.

34. Hancox RJ, Milne BJ, Taylor DR, Greene JM, Cowan JO, Flannery EM, et al. Relationship between socoioeconomic status and asthma: a longitudinal cohort study. Thorax. 2004; 59:376-80.

35. Britto MCA, Freire EFC, Bezerra PGM, Brito RCCM, Rego JC. Baixa renda como fator de proteção contra asma em crianças e adolescentes usuários do Sistema Único de Saúde. J Bras Pneumol 2008; 34:251-5.

36. Felizola MLB, Viegas CAA, Almeida M, Ferreira F, Santos MCA. Prevalência de asma brônquica e de sintomas a ela relacionados em escolares do Distrito Federal e sua relação com o nível socioeconômico. J Bras Pneumol 2005; 31:486-91.

37. Canova C, Harris JM, Mills P, White C, Moffat S, Shread L, et al. Epidemiological measures of childhood asthma: cross-sectional and longitudinal consistency. Respir Med 2012; 106:1226-35.

38. Rede Interagencial de Informações para a Saúde. Proporção de nascidos vivos de baixo peso ao nascer - G.16. http://www.ripsa.org.br/fichasIDB/ record.php?node $=$ G. $16 \&$ lang $=$ pt \&version $=$ ed 5 (acessado em 20/Out/2012).

39. Francisco PMSB, Donalísio MR, Barros MBA, César CLG, Carandina L, Goldbaum M. Medidas de associação em estudo transversal com delineamento complexo: razão de chances e razão de prevalência. Rev Bras Epidemiol 2008; 11:347-55.

40. Rumel D. "Odds ratio": algumas considerações. Rev Saúde Pública 1986; 20:253-8. 
41. Weinmayr G, Weiland SK, Björkstén B, Brunekreef B, Büchele G, Cookson WO, et al. Atopic sensitization and the international variation of asthma symptom prevalence in children. Am J Respir Crit Care Med 2007; 176:565-74.

42. McCormack MC, Breysse PN, Matsui EC, Hansel NN, Willians D, Curtin-Brosnan J, et al. Inhome particle concentrations and childhood asthma morbidity. Environ Health Perspect 2009; 117:294-8.

43. Moncayo AL, Vaca M, Oviedo G, Erazo S, Quinzo I, Fiaccone RL, et al. Risk factors for atopic and nonatopic asthma in a rural area of Ecuador. Thorax 2010; 65:409-16.
44. Silva PRS, Rosa AM, Hacon SS, Ignotti E. Hospitalização de crianças por asma na Amazônia brasileira: tendência e distribuição espacial. J Pediatr (Rio J.) 2009; 85:541-6.

45. Saldanha CT, Botelho C. Perfil de atendimento em crianças menores de cinco anos de idade com asma/sibilos em um hospital público. Rev Bras Alergia Imunopatol 2010; 33:235-40.

Recebido em 25/Jul/2012

Versão final reapresentada em 27/Mar/2013

Aprovado em 17/Abr/2013 